\title{
Association of adiponectin gene 276G > T polymorphism with polycystic ovary syndrome in Pakistani women.
}

\author{
Waqas Ahmed $^{1 *}$, Rabia Tasleem ${ }^{*}$, Shaheen Shehzad ${ }^{2}$ \\ ${ }^{1}$ Department of Bioscience, Comsats University, Islamabad, Pakistan \\ ${ }^{2}$ Department of Bioinformatics and Biotechnology, International Islamic University, Islamabad, Pakistan
}

\begin{abstract}
Objective: To investigate whether there is association of Adiponectin gene $276 \mathrm{G}>\mathrm{T}$ polymorphism with the risks of polycystic ovary syndrome (PCOS) in selected Pakistani population.

Study design: This study included samples from 106 study subjects, out of which 74 were diagnosed with PCOS and 34 were normal controls). The biochemical, Physical and clinical parameters which are assayed for the samples included menstrual problem, Past and present medical and family history, BMI, waist and hip ratio, LH, FSH, TSH and pro-lactin. Single nucleotide polymorphisms of the adiponectin gene 276 (G>T) were analysed using a PCR-RFLP method. Determination of allelic and genotypic variation with the PCOS was carried out by statistical analysis.

Results: Results showed no association of adiponectin gene $276 \mathrm{G}>\mathrm{T}$ polymorphism with the polycystic ovary syndrome (PCOS) because non-significant difference was found between distributions of genotypes and alleles of both polymorphisms in women with PCOS and controls. Levels of LH, FSH, TSH and prolactin were also analysed, and the results showed significant decline in FSH levels and increase in LH levels in PCOS females comparing with controls. A slightly significant increase was also observed in the serum levels of TSH and prolactin in PCOS women when compared with control.

Conclusion: Study suggested that obesity plays an important role in hormonal disturbances that are associated with PCOS. Polymorphism at +276 position of adiponectin gene is not associated with the PCOS and no significant link was observed.
\end{abstract}

Keywords: Polycystic ovary syndrome (PCOS), Adiponectin, Gene polymorphism, Endocrine hormones, Association study.

Accepted on December 20, 2018

\section{Introduction}

Polycystic ovary syndrome (PCOS) is known to be affecting about 5 to 10 percent of child bearing age women and adolescent girls [1]. This disorder was first defined in 1935 by Stein and Leventhal with the characteristics of hyperandrogenism, dysfunction of the ovary, polycystic ovaries, and subfertility. The onset of PCOS usually starts at puberty or soon after it and is a condition which is known to be life-long [2]. PCOS is a hormonal disorder with a potential to lead to various diseases. Signs and symptoms can include oligomenorrhea, infertility, hirsutism on face, thighs, tummy and nipples, moderate to severe acne, male pattern alopecia, sleep apnea, obesity and skin tags [3]. The genetics and imbalances in hormones level may play a role but still the exact cause of PCOS is unknown. The probability of PCOS in women is most likely to occur if the sister or mother is suffering from the condition. Insulin and increase production of androgen hormone can be other factors contributing which can cause PCOS [4].
Adiponectin (APN) is the most abundant protein which is secreted in the adipose tissue exclusively and also referred to as ACRP30 or AdipoQ [5]. Adiponectin consists of four distinct regions and is a long polypeptide made up of 244 amino acids [6]. The adiponectin gene is composed of two introns and three exons spanning about 17 kilobase pairs region [7]. The major role of adiponectin is the modulation of glucose uptake by liver and muscles, increasing fatty acid oxidation in muscles and repression of liver gluconeogenesis. As a result, adiponectin causes reduction in triglyceride a is to increase insulin sensitivity by stimulating glucose uptake in the liver and muscle, decreasing hepatic gluconeogenesis, and promoting fatty acid beta-oxidation in the skeletal muscle. Consequently, APN reduces accumulation of triglyceride (TG) accumulation and increase in insulin sensitivity [5].

Adiponectin has been known to have a possible role in PCOS by regulating steroidogenesis [8]. Two common polymorphisms were mainly under genetic association studies in the adiponectin gene, one polymorphism of the G276T position in intron 2 and one position is T45G position of exon 
2. Both the polymorphisms are reported to be associated with increased risk of obesity, insulin resistance and Diabetes mellitus type 2 [9-11]. Different studied carried out to analyse the association of polymorphism in adiponectin gene with the risk of PCOS and the results showed diversity in the association results [12,13]. The selection of these two polymorphisms is made due to their higher frequency in all the selected populations and all the other populations were reported to be very rare [14].

The objective of this study was to investigate whether there is association of adiponectin gene $276 \mathrm{G}>\mathrm{T}$ polymorphism with the risks of polycystic ovary syndrome (PCOS) in selected Pakistani population. The effect of this polymorphism on the metabolic changes and its association with the PCOS has been investigated.

\section{Materials and Methods}

\section{Study population}

The study population consisted of 200 Pakistani women (110 with PCOS and 90 normal females). The women were examined and interviewed about the sign and symptoms of PCOS. PCOS patients were recruited from the outpatient department of Gynaecology Unit 1 of Holy Family Hospital, Rawalpindi from February 2017 to May 2017. PCOS patients were evaluated by their personal information, clinical and laboratory investigation including menstrual problem, age of onset of syndrome, past and present medical and family history. General physical examination especially their height and weight for body mass index (BMI), waist and hip ratio and any other abnormality was also recorded through properly designed questionnaires (Annex I). Equal number of age and sex matched health control subjects were also included in the study. A detailed Performa was designed to record the past and present gynecological events of each subject. Anthropometric measurements including age, height and weight were recorded for the determination of BMI of PCOS and controls. The BMI of each subject was evaluated as weight $(\mathrm{kg}) / \mathrm{height}\left(\mathrm{m}^{2}\right)$.

The PCOS women were further divided in to two subgroups based on BMI. Group 1 consisted of 32 Lean PCOS women $\left(\mathrm{BMI}<25 \mathrm{~kg} / \mathrm{m}^{2}\right)$ and group 2 consisted of 78 obese PCOS $\left(\right.$ BMI $\left.>25 \mathrm{~kg} / \mathrm{m}^{2}\right)$. A group of 90 controls were also analysed for the distribution of adiponectin gene. The control group consisted of university students and hospital staff were also divided in to subgroups based on BMI.

\section{Biochemical analysis}

Serum levels of all reproductive hormones including luteinizing hormone ( $\mathrm{LH})$, follicle-stimulating hormone (FSH), Thyroid stimulating hormone (TSH) and Prolactin levels of all study subjects were analysed after drawing of blood samples using commercially available kits. According to manufacturer protocol, cobas e411 analyzer (Roche) was used for estimation of serum level of all hormones on the principle of chemiluminescence.

\section{Genotype analysis}

Using phenol-chloroform extraction method, genomic DNA was extracted rapidly from the patients and control blood samples. The genotyping of adiponectin $276 \mathrm{G}>\mathrm{T}$ polymorphism was done by PCR-RFLP using the following primers: forward, 5-GGCCTCTTTCATCACAGACC-3'; reverse 5'-AGATGCAGCAAAGCCAAAGT-3'. The amplification conditions were as follows: $95^{\circ} \mathrm{C}$ for $5 \mathrm{~min}$, followed by 35 cycles of $45 \mathrm{~s}$ at $95^{\circ} \mathrm{C}, 45 \mathrm{~s}$ at $56^{\circ} \mathrm{C}$ and $45 \mathrm{~s}$ at $72^{\circ} \mathrm{C}$, and the last step ending with $10 \mathrm{~min}$ extension step at $72^{\circ} \mathrm{C}$. The After amplification, the product was restricted using BsmI restriction enzyme using the conditions given in the manual. The digested products were separated on $2 \%$ agarose gel that was pre-stained with ethidium bromide.

\section{Statistical analysis}

Statistical analyses were done using the Statistical Package for the Social Sciences (SPSS software, version 20.0). The mean and standard deviation was calculated after the organization of data unless otherwise stated. The independent student t-test was used to compare the means of categorical variables of PCOS and controls which were described as percentages. $\chi^{2}$ test was used to evaluate the difference in categorical characteristics. The chi-square test was also used to compare the distributions of genotypes and alleles between the study groups. The constancy of genetic variation in a population of G276T polymorphisms was determined using Hardy-Weinberg equilibrium. The odds ratios (ORs) and 95\% confidence intervals (CIs) were also calculated in statistically different genotype frequencies. $\mathrm{P}$ value less than 0.05 was considered to be as statistically significant whereas $p$ value greater than 0.05 taken as non-significant.

\section{Results}

\section{Categorical variables of the study groups}

The results of categorical variables of PCOS $(n=110)$ and Controls $(n=90)$ are presented are in the Table 1 which was collected using a self-design questionnaire. The PCOS patients had a higher percentage of family history $(20.86 \%)$ as compared to the controls $(8.76 \%)$. Nearly, $31.94 \%$ of the PCOS had acne and $41.67 \%$ had an oily and the percentage was decreased in the controls (31.94 and 41.67\%). A very little percentage of the study subject had complaints of galactorrhea $(1.39 \%)$. A significantly higher percentage of hirsutism, acanthosis nigrican and infertility were observed in the subjects with PCOS and details are listed in the Table 1.

\section{Anthropometric and endocrine data of study group}

Anthropometric parameters and endocrine data of the population studied are summarized in Table 2. Mean age of the study group with PCOS was $27.39 \pm 5.209$ and $28.80 \pm 5.38$ in the control group. A significant difference in BMI was recorded in the PCOS women when compared with the control group (27.06 \pm 6.12 vs. $24.63 \pm 3.07 ; \mathrm{P}=0.001)$. In comparing 
BMI matched groups, BMI of the lean PCOS was not found to be significantly different from the lean controls $(22.44 \pm 2.75$ vs. $22.05 \pm 1.98 ; \mathrm{P}=0.456)$ while $\mathrm{BMI}$ was significantly higher in Obese PCOS in comparison to obese controls $(31.68 \pm 4.40$ vs. $27.21 \pm 1.20 ; \mathrm{P}=0.001)$. $\mathrm{LH}$ was found to have significantly increased serum level in the PCOS group when compared with the normal women $(17.34 \pm 3.43$ vs. $9.02 \pm 3.11 ; \mathrm{P}=0.02)$ and increased level of $\mathrm{LH}$ results was also recorded in PCOS women when comparing BMI matched groups listed in Table 2. No significant difference was observed in the in serum FSH levels in all the study groups. TSH level was not significantly different in PCOS patients when compared to the controls $(1.65 \pm 1.14$ vs. $1.30 \pm 1.1 ; \mathrm{P}=0.299)$. On comparison the level of serum TSH within PCOS, the obese patients have higher levels of TSH $(1.78 \pm 1.42$ vs. $1.53 \pm 1.65 ; \mathrm{P}=0.006)$. As compared to control women group, prolactin serum level was significantly elevated in PCOS women study groups $(\mathrm{P}=0.05)$.

\section{Genotype analysis and association of gene polymorphism with PCOS}

The genotyping for adiponectin $276 \mathrm{G}>\mathrm{T}$ was done for polycystic ovary syndrome patients along with healthy controls. Distribution of genotype of $276 \mathrm{G}>\mathrm{T}$ adiponectin gene polymorphism were in the Hardy-Weinberg equilibrium $(\mathrm{P}>0.05)$ in both the control and PCOS group. The homozygous GG genotype was slightly higher in patients as compared to controls. The difference in genotype and allelic distribution for $276 \mathrm{G}>\mathrm{T}$ polymorphism was found to be statistically insignificant for both control and PCOS women. Overall the results indicated that polymorphism at position G276T was not associated with increased risk of PCOS and the details are listed in Table 3.

\section{Distribution of 276G $>$ T gene polymorphisms in PCOS women according to $B M I$}

The allelic and genotypic frequencies of the two PCOS women subgroups which were divided on the basis of BMI are shown in Table 4. Homozygous GG genotype and G allele at position +276 T allele were higher in obese PCOS women as compared to normal weight PCOS women and the GT genotype and T allele were little higher among obese PCOS women than in normal weight PCOS women, but the differences in the distribution were statistically non-significant $(\mathrm{P}<0.05$; $\mathrm{OR}=0.958 ; 95 \% \quad \mathrm{CI}, 0.368-2.585)$. Results showed that genotypic and allelic gene polymorphism frequency between the two PCOS group based on BMI were not statistically different as shown in Table 4.

Table 1. Categorical variables in both the study groups.

\begin{tabular}{lll}
\hline Variables (\%) & PCOS $(\mathbf{n = 1 1 0 )}$ & Control $(\mathbf{n = 9 0 )}$ \\
\hline Family history & 20.83 & 8.76 \\
\hline Married & 87.8 & 59.2 \\
\hline Acne & 31.94 & 16.9 \\
\hline Oily skin & 41.67 & 20.56 \\
\hline Hirsutism & 66.67 & 14.39 \\
\hline Irregular menstrual periods & 83.33 & 30.89 \\
\hline Acanthosis nigrican & 37.78 & 13.56 \\
\hline Glactorrhea & 1.39 & 2.3 \\
\hline Obesity & 70.9 & 42.22 \\
\hline Infertility & 47.22 & 19.06 \\
\hline
\end{tabular}

Table 2. Biochemical and clinical parameters of the study groups.

\begin{tabular}{|c|c|c|c|c|c|c|}
\hline Parameters & $\operatorname{PCOS}(n=110)$ & Controls $(n=90)$ & Lean PCos $(n=32)$ & Lean controls $(n=52)$ & Obese PCOS $(n=78)$ & Obese controls $(n=38)$ \\
\hline Age & $27.39 \pm 5.209$ & $28.80 \pm 5.38$ & $26.71 \pm 3.26$ & $28.90 \pm 5.67$ & $28.06 \pm 5.73$ & $28.71 \pm 5.05$ \\
\hline BMI $\left(\mathrm{kg} / \mathrm{m}^{2}\right)$ & $27.06 \pm 6.12$ & $24.63 \pm 3.07$ & $22.44 \pm 2.75$ & $22.05 \pm 1.98$ & $31.68 \pm 4.40$ & $27.21 \pm 1.20$ \\
\hline FSH (miu/ml) & $5.62 \pm 2.35$ & $6.20 \pm 3.3$ & $5.72 \pm 1.78$ & $6.23 \pm 1.7$ & $5.55 \pm 2.32$ & $6.18 \pm 18$ \\
\hline $\mathrm{LH}(\mathrm{miu} / \mathrm{ml})$ & $17.34 \pm 3.43$ & $9.02 \pm 3.11$ & $17.13 \pm 2.67$ & $8.87 \pm 2.78$ & $17.56 \pm 1.17$ & $9.17 \pm 3.21$ \\
\hline TSH (miu/ml) & $1.65 \pm 1.14$ & $1.30 \pm 1.1$ & $1.53 \pm 1.65$ & $1.20 \pm 0.91$ & $1.78 \pm 1.42$ & $1.40 \pm 2.09$ \\
\hline Prolactin (miu/L) & $612 \pm 17.34$ & $132.2 \pm 12.3$ & $594 \pm 15.64$ & $122.2 \pm 11.2$ & $630 \pm 16.34$ & $142 \pm 13.2$ \\
\hline
\end{tabular}

Data are represented as mean \pm SD; PCOS vs. controls $\mathrm{P}<0.05$; obese PCOS vs. lean PCOS P<0.05; obese PCOS vs. obese normal $\mathrm{P}<0.05$; lean PCOS vs. lean controls $\mathrm{P}<0.05$.

Table 3. Genotype and allele frequencies of $276 G>T$ polymorphisms in the PCOS and controls.

\begin{tabular}{lllll}
\hline Polymorphism & PCOS $(\mathbf{n = 1 1 0})$ & Controls $(\mathbf{n = 9 0 )}$ & P-value & Odds ratio \\
\hline Genotypes, $n(\%)$ & & & \\
\hline GG & $57(51.81)$ & $48(53.3)$ & 0.977 & 0.976 \\
\hline TT & $10(11.11)$ & $8(8.88)$ & $0.368-2.585$ & \\
\hline
\end{tabular}




\begin{tabular}{|c|c|c|c|c|c|}
\hline GT & $43(39.09)$ & $34(37.78)$ & & & \\
\hline \multicolumn{6}{|c|}{ Allele, n (\%) } \\
\hline G & $157(71.36)$ & $130(72.2)$ & 0.911 & 0.958 & $0.619-1.485$ \\
\hline $\mathrm{T}$ & $63(28.64)$ & $50(27.8)$ & & & \\
\hline
\end{tabular}

Data are presented as mean \pm SD. "Statistically significant difference was determined between groups $(P<0.05)$.

Table 4. Genotype and allele frequencies of $276 G>T$ polymorphisms in two PCOS study groups.

\begin{tabular}{|c|c|c|c|c|c|}
\hline & Group $1(n=34)$ & Group $2(n=76)$ & P-value & Odds ratio & C.I. (95\%) \\
\hline \multicolumn{6}{|c|}{ Polymorphism } \\
\hline \multicolumn{6}{|c|}{ Genotypes, n (\%) } \\
\hline GG & $14(41.17)$ & $43(56.57)$ & & & \\
\hline TT & $3(8.82)$ & $7(9.21)$ & 0.276 & 1.048 & $0.245-4.325$ \\
\hline GT & $17(50.0)$ & $26(34.21)$ & & & \\
\hline \multicolumn{6}{|c|}{ Allele, n (\%) } \\
\hline G & $45(66.17)$ & $112(73.68)$ & 0.263 & 1.431 & $0.771-2.657$ \\
\hline $\mathrm{T}$ & $23(33.82)$ & $40(26.31)$ & & & \\
\hline
\end{tabular}

\section{Discussion}

In this study we investigated whether the polymorphism in adiponectin gene at position +276 is associated with risk of PCOS in Pakistani selected population and whether the obesity is associated with increased risk of PCOS. The endocrine and clinical parameters and its association with the PCOS were also determined in the selected population.

Hormonal estimation is known to be the main diagnostic marker of PCOS over the years. That's why in the present study the serum level of some hormones was estimated of the study group. In a previous study an elevated level of LH was observed among PCOS women as compared to normal women [15]. However, in present study the level of LH was significantly elevated but not intense. LH levels was same in PCOS sub groups based on BMI that means that obesity was not found to have any role in increasing or decreasing LH level as reported earlier in South Indian women [16]. It is suggested that there might be some link between BMI and LH [8]. No association was observed in the serum FSH level in PCOS women as compared to controls. Elevated LH level can be important factor in diagnosis of PCOS.

Serum TSH levels in the study subject was not significantly differentiated between PCOS and control. As compared to normal control group, Prolactin level was significantly increased in PCOS patients $(p=0.006)$. our results are in agreement and confirmed with already reported previous studies carried out, in which significantly elevated prolactin levels was observed in PCOS women as compared to control [15]. In a study higher prolactin level was observed as compared to normal control in agreement to our study [17]. On the other hand, in disagreement with our results reported that, increased level of prolactin is not associated with PCOS and is not a clinical manifestation for diagnosis of PCOS [18]. Swathi et al. in disagreement with our observations concluded that hyperprolactinemia and PCOS are two different entities [19].

In the present study, the association was analysed between the SNP $+276(\mathrm{G} / \mathrm{T})$ in the adiponectin gene and PCOS women. No association was found between the SNP $+276(\mathrm{G} / \mathrm{T})$ and PCOS in selected Pakistani women population. Variation in the adiponectin gene determines the phenotype overexpression of the metabolic syndrome to definite extent. However, a disagreement with our result has been reported that there is association between the SNPs $+276(\mathrm{G} / \mathrm{T})$ in the adiponectin gene and PCOS in Korean women [13]. But the effect of this polymorphism on the expression of adiponectin gene is not fully understood. According to Xita, a total 13 SNPs in the adiponectin gene have been identified, in which the focus of study was on the two SNPS $45(\mathrm{~T} \rightarrow \mathrm{G})$ and $276(\mathrm{G} \rightarrow \mathrm{T})$. [20]. In agreement with the results, the analysis carried out in the south Indian women reported that PCOS susceptibility is not associated with polymorphism in adiponectin gene [16]. A group of Greek PCOS women was also investigated and no association was found in gene polymorphism at position +45 and +276 with the risk of PCOS [20].

On the other hand, it was observed that the percentage of GG and GT genotype at position +276 of the adiponectin gene was little higher in Obese PCOS (BMI>25) as compared to lean PCOS women $(\mathrm{BMI}<25)$ while the percentage of TT genotype was almost same in both the sub groups but the difference was 
not statistically significant. In a study the GT genotype of $276 \mathrm{G}>\mathrm{T}$ polymorphism was more frequent in the obese PCOS group and the homozygous TT distribution was not different in both the groups [21]. No significant difference in allelic frequency for adiponectin SNP +276 was found between the two PCOS sub groups.

\section{Conclusion}

In conclusion the criteria for the diagnosis of PCOS on the basis of endocrine hormonal study can be reinforced. Mainly LH and prolactin levels can play important role in early diagnosis of the syndrome. Our results checked the association of one SNP of the adiponectin gene with PCOS patients in Pakistani population. The adiponectin gene doesn't seem to have a causative role in the PCOS development. The outcomes of our results provided important basis for the early diagnosis and prevention of PCOS.

\section{Acknowledgement}

Authors express their deepest gratitude to Director General Dr. Ismail, Institute of Biomedical and Genetic Engineering, KRL hospital Islamabad, who gave us access to the laboratory and research facilities. Authors thank the staff of department of Gynecology Unit 1 of Holy Family Hospital, Rawalpindi Hospital for their help.

\section{Ethics}

This study was approved by the Ethical Review Committee of International Islamic University Islamabad. Written informed consent was obtained from all study participants after informing them about the objectives of study.

\section{Consent for Publication}

I confirm that all authors agree on publication and give its liability to the corresponding author.

\section{Competing Interests}

The authors declare that they have no competing interests. The authors also declare not to have any financial support. Also the work-done was financially independent.

\section{References}

1. Scalzo K, McKittrick M. Case problem: dietary recommendations to combat obesity, insulin resistance, and other concerns related to polycystic ovary syndrome. J Am Diet Assoc 2000; 100: 955-957.

2. Kitzinger $\mathrm{C}$, Willmott J. The thief of womanhood: womens experience of polycystic ovarian syndrome. Soc Sci Med 2002; 54: 349-361.

3. Sheehan MT. Polycystic ovarian syndrome: diagnosis and management. Clin Med Res 2004; 2: 13-27.

4. Miller MDK, Nixon C, Boots LR, Go RC, Azziz R. Prevalence of polycystic ovary syndrome (PCOS) in first- degree relatives of patients with PCOS. Fertil Steril 2001; 75: 1 .

5. Bohler H, Mokshagundam S, Winters SJ. Adipose tissue and reproduction in women. Fertil Steril 2010; 94: 795-825.

6. Shapiro L, Scherer PE. The crystal structure of a complement-1q family protein suggests an evolutionary link to tumor necrosis factor. Curr Biol 1998; 8: 335-338.

7. Takahashi M, Arita Y, Yamagata K, Matsukawa Y, Okutomi K, Horie M, Shimomura I, Hotta K, Kuriyama H, Kihara S, Nakamura T, Yamashita S, Funahashi T, Matsuzawa Y. Genomic structure and mutations in adipose-specific gene, adiponectin. Int $\mathrm{J}$ Obes Relat Metab Disord 2000; 24: 861-868.

8. Panidis D, Kourtis A, Farmakiotis D, Mouslech T, Rousso D, Koliakos G. Serum adiponectin levels in women with polycystic ovary syndrome. Hum Reprod 2003; 18: 1790-1796.

9. Filippi E, Sentinelli F, Trischitta V, Romeo S, Arca M, Leonetti F, Di Mario U, Baroni MG. Association of the human adiponectin gene and insulin resistance. Eur J Hum Genet 2004; 12: 199-205.

10. Hwang JY, Park JE, Choi YJ, Huh KB, Kim WY. SNP276G $>$ T polymorphism in the adiponectin gene is associated with metabolic syndrome in patients with type II diabetes mellitus in Korea. Eur J Clin Nutr 2010; 64: 105-107.

11. Xian L, He W, Pang F, Hu Y. ADIPOQ gene polymorphisms and susceptibility to polycystic ovary syndrome: a HuGE survey and meta-analysis. Eur J Obstet Gynecol Reprod Biol 2012; 161: 117-124.

12. Heinonen S, Korhonen S, Helisalmi S, Koivunen R, Tapanainen JS, Hippelainen MI. Associations between two single nucleotide polymorphisms in the adiponectin gene and polycystic ovary syndrome. Gynecol Endocrinol 2005; 21: 165-169.

13. Li L, Yun JH, Lee JH, Song S, Choi BC, Baek KH. Association study of $+45 \mathrm{G} 15 \mathrm{G}(\mathrm{T} / \mathrm{G})$ and $+276(\mathrm{G} / \mathrm{T})$ polymorphisms in the adiponectin gene in patients with polycystic ovary syndrome. Int J Mol Med 2011; 27: 283-287.

14. Panidis D, Kourtis A, Kukuvitis A, Farmakiotis D, Xita $\mathrm{N}$, Georgiou I, Tsatsoulis A. Association of the T45G polymorphism in exon 2 of the adiponectin gene with polycystic ovary syndrome: role of Delta4androstenedione. Hum Reprod 2004; 19: 1728-1733.

15. Akram M, Roohi N. Endocrine correlates of polycystic ovary syndrome in Pakistani women. J Coll Physicians Surg Pak 2015; 25: 22-26.

16. Nambiar V, Vijesh VV, Lakshmanan P, Sukumaran S, Suganthi R. Association of adiponectin and resistin gene polymorphisms in south indian women with polycystic ovary syndrome. Eur J Obstet Gynecol Reprod Biol 2016; 200: $82-88$. 
17. Esmaeilzadeh S, Mirabi P, Andarieh MG. The serum prolactin level in infertile women with polycystic ovary syndrome. J Babol Univ Med Sci 2014; 16: 63-68.

18. Filho RB, Domingues L, Naves L, Ferraz E, Alves A, Casulari LA. Polycystic ovary syndrome and hyperprolactinemia are distinct entities. Gynecol Endocrinol 2007; 23: 267-272.

19. Swathi T, Sujaya VR. Prevalance of Hyperprolactinemia in PCOS. Int J Sci Res 2017; 6: 11.

20. Xita N, Georgiou I, Chatzikyriakidou A, Vounatsou M, Papassotiriou GP. Effect of adiponectin gene polymorphisms on circulating adiponectin and insulin resistance indexes in women with polycystic ovary syndrome. Clin Chem 2005; 51: 416-423.

21. Xita N, Georgiou I, Tsatsoulis A, Kourtis A, Kukuvitis A, Panidis D. A polymorphism in the resistin gene promoter is associated with body mass index in women with polycystic ovary syndrome. Fertil Steril 2004; 82: 1466-1467.

\section{*Correspondence to}

Waqas Ahmed

Department of Bioscience

Comsats University

Islamabad

Pakistan 